\title{
Visit-to-visit variability in estimated glomerular filtration rate predicts hospitalization and death due to cardiovascular events
}

\author{
Akira Suzuki $^{1} \cdot$ Yoshitsugu Obi $^{2} \cdot$ Terumasa Hayashi $^{3} \cdot$ Naoto Kotani $^{4} \cdot$ Yukari Uemura $^{5} \cdot$ Enyu Imai $^{6}$. \\ Hirofumi Makino ${ }^{7}$. Akira Hishida ${ }^{8}$
}

Received: 13 July 2018 / Accepted: 7 January 2019 / Published online: 28 January 2019

(c) The Author(s) 2019

\begin{abstract}
Background Greater variability in estimated glomerular filtration rate (eGFR) is associated with mortality in patients with chronic kidney disease (CKD). However, the association between eGFR variability and cardiovascular (CV) mortality and/ or end-stage kidney disease (ESKD) in the CKD population is not very clear. This study aimed to clarify whether such an association exists.

Methods We analyzed a final cohort of 2869 eligible Asian patients with CKD. Patients were stratified into three groups according to eGFR variability during the first year and were followed-up for a median of 3.15 years. Primary CV composite endpoints were hospitalization or death due to CV events, and renal composite endpoints were doubling of serum creatinine levels or ESKD. Multivariate Cox hazard models adjusted for classical risk factors and eGFR slope were used to examine the $\mathrm{CV}$ and renal risk associated with eGFR variability.

Results CV endpoints were observed in 14 (2.89\%), 25 (5.69\%), and 41 (10.79\%) patients and renal endpoints were observed in 165 (27.6\%), 235 (39.0\%), and 298 patients (50.9\%) in the lowest, intermediate, and highest tertiles of eGFR variability, respectively. Patients in the highest tertile were at a significantly higher risk for CV events (hazard ratio 1.90; 95\% confidence interval 1.03-3.71) than those in the lowest tertile. However, there was no association between eGFR variability and renal endpoints.
\end{abstract}

Conclusions Variability in eGFR can predict CV outcomes among patients with CKD.

Keywords Chronic kidney disease $\cdot$ Variability $\cdot$ Estimated glomerular filtration rate $\cdot$ Cardiovascular mortality $\cdot$ End-stage kidney disease

Akira Suzuki

suzuki-akira@osaka.jcho.go.jp

1 Department of Internal Medicine, Japan Community Healthcare Organization Osaka Hospital, 4-2-78 Fukushima Fukushima-ku, Osaka 553-0003, Japan

2 Harold Simmons Center for Kidney Disease Research and Epidemiology, University of California, Irvine, CA, USA

3 Department of Kidney Disease and Hypertension, Osaka General Medical Center, Osaka, Japan

4 Pharmaceuticals and Medical Devices Agency, Center for Product Evaluation, Biostatistics Group, Tokyo, Japan

5 Clinical Research Support Center, Biostatistics Division, The University of Tokyo Hospital, Tokyo, Japan

6 Nakayamadera Imai Clinic, Takarazuka, Hyogo, Japan

7 Okayama University, Okayama, Okayama, Japan

8 Yaizu City Hospital, Yaizu, Shizuoka, Japan

\section{Introduction}

Chronic kidney disease (CKD) is a huge public health problem that affects more than $10 \%$ of the population worldwide [1] and approximately 13.3 million in Japan [2]. Impaired kidney function is associated with incident cardiovascular disease (CVD), all-cause mortality, and end-stage kidney disease (ESKD) [3-5]. The number of patients with ESKD on chronic dialysis has continued to increase worldwide over the past few decades, and ESKD has thus emerged as a financial burden. A single estimated glomerular filtration rate (eGFR) was historically adopted as a predictive variable for ESKD in observational studies; however, recent reports have demonstrated that a decreased rate of eGFR was an independent predictor for ESKD in patients with CKD [6, 7]. Notably, a transient increase in eGFR was also associated with risks for CVD and all-cause mortality [8]. These 
findings suggest that eGFR variation may be a novel predictive factor for poorer clinical outcomes of patients with CKD. Two retrospective observational studies revealed that eGFR variability was a risk factor for CVD and allcause mortality $[9,10]$. The aim of the present study was to prospectively investigate the relationship between eGFR variability, and CVD and ESKD incidence among the CKD population.

\section{Materials and methods}

\section{Study population}

The design and method used for the Chronic Kidney Disease Japan Cohort (CKD-JAC) study have been published elsewhere [11]. Briefly, the inclusion criteria were as follows: (1) Japanese or Asian patients living in Japan; (2) age between 20 and 75 years; and (3) a broad spectrum of CKD stages, defined as an eGFR of $10-59 \mathrm{ml} / \mathrm{min} / 1.73 \mathrm{~m}^{2}$. The eGFR was calculated using the estimation equation for Japanese patients [12]. The exclusion criteria were as follows: (1) polycystic kidney disease, human immunodeficiency virus infection, liver cirrhosis, active cancer, or cancer treatment within the past 2 years; (2) history of transplant and chronic dialysis; (3) pregnancy in women; and (4) refusal to provide informed consent. Recruitment started in April 2007, and 2,966 participants were followed-up until March 2013. The median follow-up period was 3.9 years. The protocol was approved by the Ethics Committee of each participating medical institute, and all participants provided informed consent. Baseline characteristics of all patients enrolled in CKD-JAC have been described previously [13].

\section{Predictor variable}

The predictor variable was the eGFR variability during the first year of the study. The variability was defined as the absolute residual of the eGFR regression line/the expected eGFR estimated by the linear regression line at each time point. The variability for each patient was calculated as the mean value of the variability. Patients were stratified into three groups according to the tertile of the mean variability during the first year.

\section{Primary endpoints}

Two primary composite endpoints were defined: (1) hospitalization due to congestive heart failure or death due to CVD and (2) doubling of serum creatinine levels or ESKD. CVD included acute myocardial infarction, heart failure, arrhythmia, stroke, and aorta dissection. The doubling of serum creatinine levels was defined as three consecutive values of serum creatinine that were twice as high as the baseline serum creatinine.

\section{Statistical analysis}

Continuous variables were presented as mean and standard deviation or median and range, and categorical variables were presented as frequency and percentage. For missing values, we used the multiple imputation method. Differences in baseline characteristics were tested using one-way analysis of variance for continuous variables, and the Chi square test was used for categorical variables.

To test the association between time to the endpoint and the mean variability in eGFR, the Kaplan-Meier curve and Cox proportional hazard model were used for unadjusted and multivariate-adjusted analysis, respectively. Baseline covariates included in the models were age, sex, body mass index, systolic blood pressure, diastolic blood pressure, prior CVD, diabetes mellitus, use of diuretics, use of antihypertensive agents, and baseline eGFR. The variability in eGFR was included in models two and three. The eGFR slope was included in model three. $p<0.05$ was considered statistically significant. All analyses were performed using SAS software version 9.4 .

\section{Results}

A total of 2,966 patients with stage 3-5 CKD were enrolled (Fig. 1). Twenty patients were excluded due to CVD or death occurring during the first year of observation. Seventy-seven patients were excluded because their serum creatinine levels were measured two times or less during the first year. Therefore, a total of 2869 patients were eligible and were followed-up for a median of 3.15 years ( $0.05-4.34$ years). The eligible subjects were stratified into three groups according to the eGFR variability during the first year of the study period. Baseline characteristics stratified by the eGFR variability are shown in Tables 1 and 2 . The tertile with the highest eGFR variability showed significantly more instances of ischemic heart disease, stroke, and diabetes. This tertile also demonstrated significantly older age, lower diastolic blood pressure, higher serum uric acid levels, lower eGFR, lower serum albumin levels, and lower hemoglobin levels.

During follow-up, 14 cardiovascular (CV) events (2.89\%) occurred in the lowest tertile, $25(5.69 \%)$ occurred in the intermediate tertile, and $41(10.79 \%)$ occurred in the highest tertile of variability. Kaplan-Meier survival curves for $\mathrm{CV}$ events are shown in Fig. 2. The highest tertile showed significantly worse $\mathrm{CV}$ event-free survival compared to the lowest tertile ( $p<0.0001 ; \log$ rank test).

A multivariate-adjusted Cox proportional hazard model indicated that the patients in the highest tertile showed an 
Fig. 1 Patients disposition. $C V$ cardiovascular. $\mathrm{Cr}$ creatinine

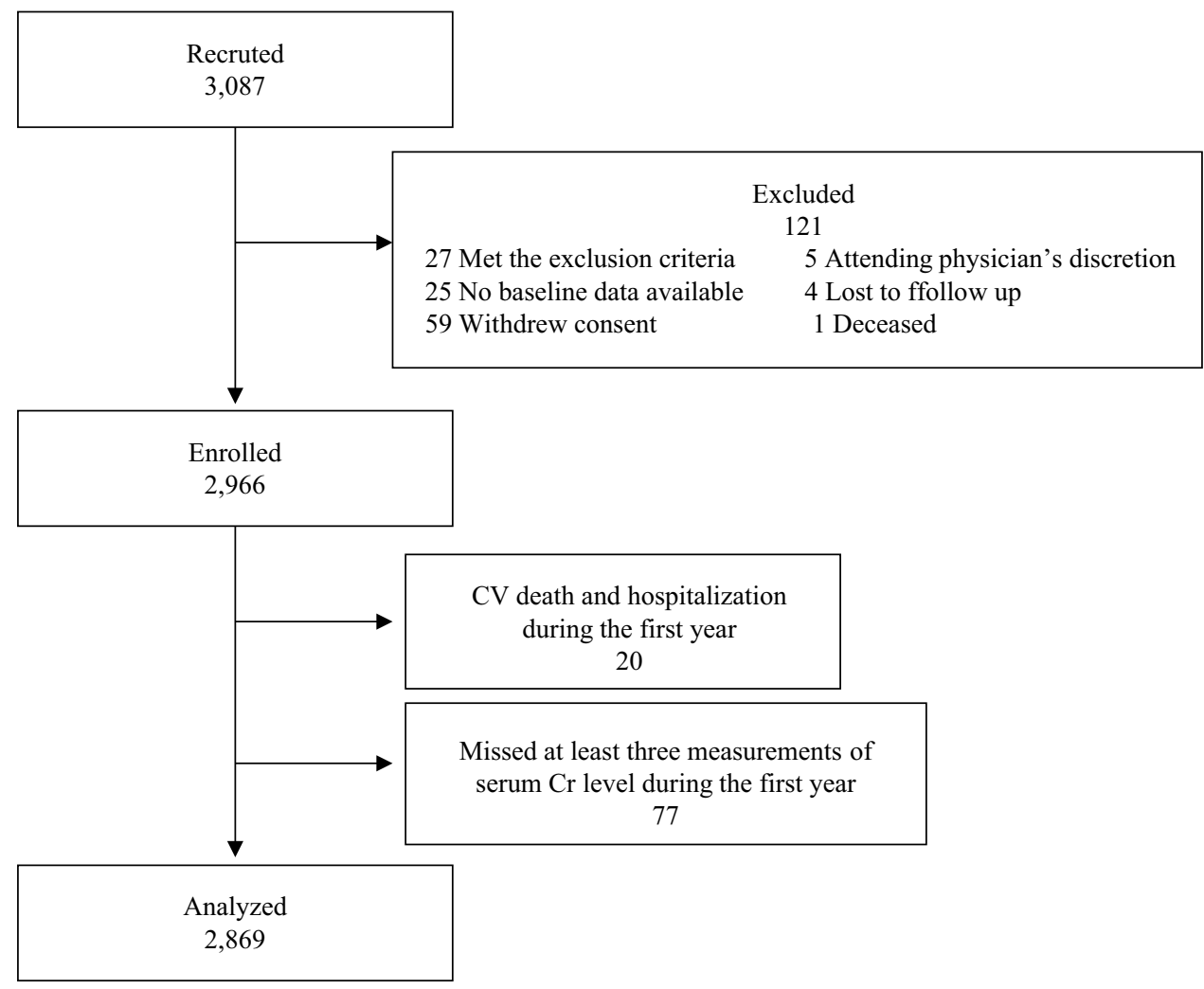

Table 1 Baseline demographic characteristics of the tertile of eGFR variability

\begin{tabular}{|c|c|c|c|c|c|c|c|}
\hline & \multicolumn{2}{|c|}{ Lowest $(n=956)$} & \multicolumn{2}{|c|}{$\begin{array}{l}\text { Intermediate } \\
(n=957)\end{array}$} & \multicolumn{2}{|c|}{ Highest $(n=956)$} & \multirow[t]{2}{*}{$p$ value } \\
\hline & $n$ & $(\%)$ & $n$ & $(\%)$ & $n$ & $(\%)$ & \\
\hline Gender (male) & 598 & 62.6 & 603 & 63.0 & 585 & 61.2 & 0.6952 \\
\hline Ischemic haeart disease & 99 & 10.4 & 120 & 12.5 & 177 & 18.5 & $<0.0001$ \\
\hline Stroke & 76 & 8.0 & 111 & 11.6 & 140 & 14.6 & $<0.0001$ \\
\hline Aorta disease or PAD & 60 & 6.3 & 71 & 7.4 & 95 & 9.9 & 0.0098 \\
\hline Diabetes mellitus & 216 & 22.6 & 314 & 32.8 & 426 & 44.6 & $<0.0001$ \\
\hline Use of diuretics & 176 & 18.41 & 267 & 27.9 & 427 & 44.67 & $<0.0001$ \\
\hline Use of antihypertensive drug & 780 & 81.6 & 779 & 81.4 & 797 & 83.37 & 0.4642 \\
\hline
\end{tabular}

$P A D$ peripheral artery disease

increased risk of $\mathrm{CV}$ events compared with those in the lowest tertile [hazard ratio (HR), 1.90; 95\% confidence interval (CI), 1.03-3.71] (Table 3). A significantly increased risk was also associated with the following variables: age (HR 1.06; 95\% CI 1.02-1.09); history of CV event (HR 2.53; 95\% CI 1.55-4.08); male sex (HR 1.86; 95\% CI 1.10-3.28); use of diuretics (HR 1.73; 95\% CI 1.06-2.85); and baseline eGFR (HR 0.97; 95\% CI 0.95-0.99).

However, 165 renal events (27.6\%) occurred in the lowest tertile, 235 (39.0\%) occurred in the intermediate tertile, and 298 (50.9\%) occurred in the highest tertile of variability. Although the Kaplan-Meier survival curve for renal events revealed a significant association between the eGFR variability and the time for renal events (Fig. 3), the association was not observed after adjustment for the eGFR slope in model three (Table 4).

\section{Discussion}

The CKD-JAC study was a multicenter, prospective cohort study that recruited pre-dialysis CKD Japanese patients. In this setting, variability in eGFR was associated with an increased risk of CV hospitalization or death, even after adjustment for classical risk factors and a decreased rate of eGFR. Patients in the highest tertile of variability 


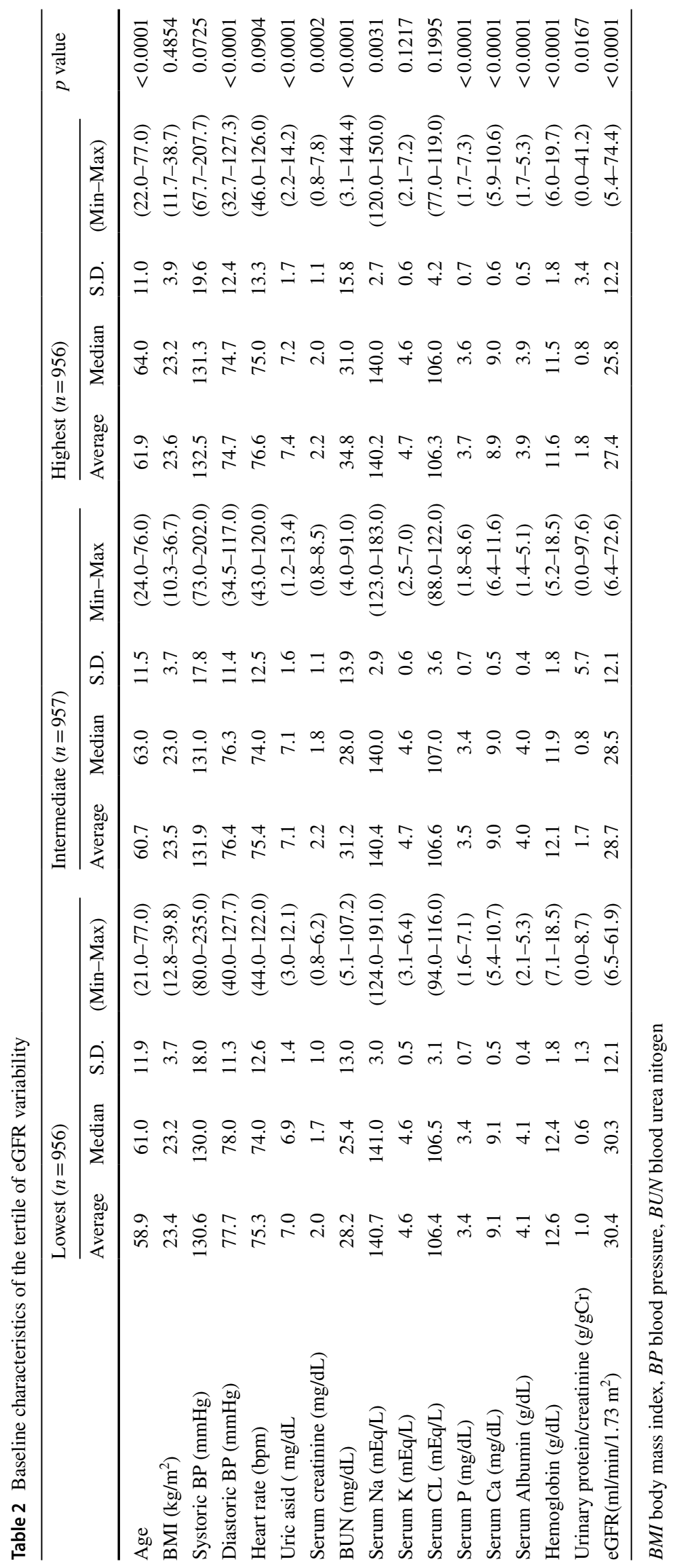




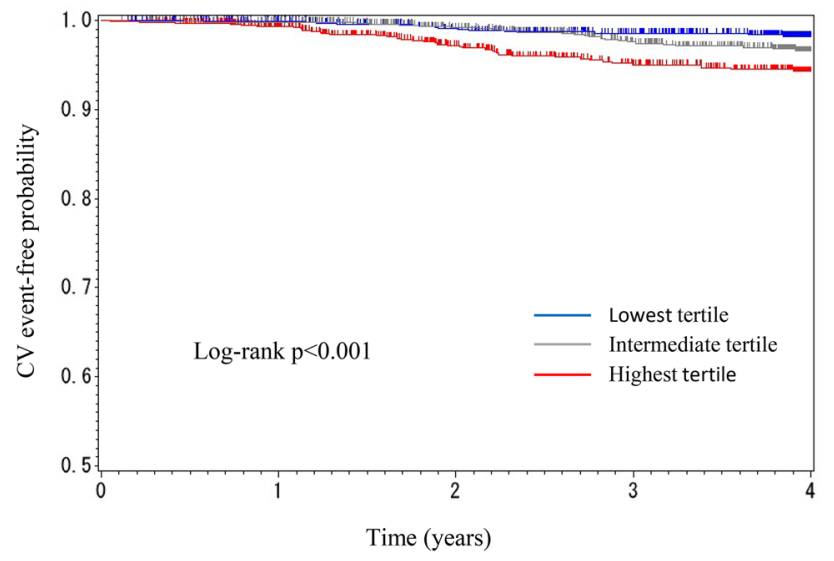

Fig. 2 Kaplan-Meier curve (time to CV event) by tertile of eGFR variability

demonstrated a $90 \%$ increased risk compared with those in the lowest tertile, whereas baseline eGFR only had a modest association and the decreased rate of eGFR had no significant association with $\mathrm{CV}$ events. CKD is one of the established risk factors for CVD. Therefore, eGFR variability may provide more powerful prognostic information for CVD than baseline eGFR and eGFR slope.

Although the variation in eGFR can be generated by hemodynamic changes, the risk of variability in eGFR was independent of blood pressure and use of antihypertensive drugs or diuretics. The highest variability tertile comprised patients with a history of ischemic heart disease, stroke, and diabetes; however, the association between $\mathrm{CV}$ risk and variability in eGFR was significant after adjustment for these comorbidities. The highest tertile also demonstrated older age, lower diastolic blood pressure, higher serum uric acid levels, higher serum creatinine levels, higher blood urea nitrogen levels, higher serum Na levels, higher serum phosphate levels, lower serum Ca levels, lower serum albumin levels, lower $\mathrm{Hb}$ levels, and higher urinary protein levels. It was not possible to adjust the multivariate analysis by urinary protein due to the many missing values (deficit rate $67.4 \%)$.

Two recent retrospective cohort studies revealed that the variability in eGFR was independently associated with all-cause mortality for CKD patients [9, 10]. They defined the variability as the coefficient of variation of the regression line coefficient of eGFR, or as the absolute value of the residual of the eGFR regression line. The definition of variability in eGFR in the present study was the absolute residual of the eGFR regression line/the expected eGFR estimated by the linear regression line at each time point, which was modified from the definition indicated by Perkins. This parameter should be more accurate because it is independent of the eGFR value. Further studies are needed to evaluate the
Table 3 Multivariate-adjusted Cox proportional hazard for CV events

\begin{tabular}{|c|c|c|c|}
\hline & H.R. & 95\% C.I. & $p$ value \\
\hline \multicolumn{4}{|l|}{ Model 1} \\
\hline Gender (male vs female) & 1.85 & {$[1.10,3.26]$} & 0.0260 \\
\hline Age (1-year increase) & 1.06 & {$[1.03,1.09]$} & 0.0008 \\
\hline BMI & 1.02 & {$[0.95,1.09]$} & 0.6018 \\
\hline History of heart disease & 2.60 & {$[1.60,4.19]$} & $<0.0001$ \\
\hline Diabetes Mellitus & 1.20 & {$[0.72,2.00]$} & 0.4953 \\
\hline Diastoric BP & 0.98 & {$[0.96,1.01]$} & 0.1334 \\
\hline Systoric BP & 1.01 & {$[1.00,1.03]$} & 0.1536 \\
\hline Use of diuretics & 1.86 & {$[1.14,3.05]$} & 0.0127 \\
\hline Use of ACE & 0.94 & {$[0.57,1.51]$} & 0.7970 \\
\hline eGFR $\left(1 \mathrm{ml} / \mathrm{min} / 1.73 \mathrm{~m}^{2}\right.$ increse $)$ & 0.97 & {$[0.95,0.99]$} & 0.0032 \\
\hline \multicolumn{4}{|l|}{ Model 2} \\
\hline $\begin{array}{l}\text { eGFR variability (intermediate vs } \\
\text { lowest) }\end{array}$ & 1.41 & {$[0.74,2.80]$} & 0.3083 \\
\hline (Highest vs lowest) & 1.92 & {$[1.04,3.75]$} & 0.0429 \\
\hline Gender (male vs female) & 1.86 & {$[1.11,3.29]$} & 0.0243 \\
\hline Age (1-year increase) & 1.06 & {$[1.02,1.09]$} & 0.0009 \\
\hline BMI & 1.02 & {$[0.95,1.09]$} & 0.6266 \\
\hline History of heart disease & 2.52 & {$[1.55,4.06]$} & 0.0002 \\
\hline Diabeyes Mellitus & 1.14 & {$[0.68,1.91]$} & 0.6105 \\
\hline Diastoric BP & 0.98 & {$[0.96,1.01]$} & 0.1748 \\
\hline Systoric BP & 1.01 & {$[1.00,1.03]$} & 0.1727 \\
\hline Use of diuretics & 1.72 & {$[1.05,2.83]$} & 0.0321 \\
\hline Use of ACE & 0.90 & {$[0.54,1.45]$} & 0.6735 \\
\hline eGFR ( $1 \mathrm{ml} / \mathrm{min} / 1.73 \mathrm{~m}^{2}$ increse $)$ & 0.97 & {$[0.95,0.99]$} & 0.0038 \\
\hline \multicolumn{4}{|l|}{ Model 3} \\
\hline $\begin{array}{l}\text { eGFR variability (Intermediate vs } \\
\text { lowest) }\end{array}$ & 1.41 & {$[0.74,2.80]$} & 0.3089 \\
\hline (Highest vs lowest) & 1.90 & {$[1.03,3.71]$} & 0.0469 \\
\hline Gender (male vs female) & 1.86 & {$[1.10,3.28]$} & 0.0248 \\
\hline Age(1-year increase) & 1.06 & {$[1.02,1.09]$} & 0.0009 \\
\hline BMI & 1.02 & {$[0.95,1.09]$} & 0.6296 \\
\hline History of heart disease & 2.53 & {$[1.55,4.08]$} & 0.0002 \\
\hline Diabetes & 1.12 & {$[0.67,1.89]$} & 0.6603 \\
\hline Diastoric BP & 0.98 & {$[0.96,1.01]$} & 0.1736 \\
\hline Systoric BP & 1.01 & {$[0.99,1.03]$} & 0.1988 \\
\hline Use of diuretics & 1.73 & {$[1.06,2.85]$} & 0.0296 \\
\hline Use of ACE & 0.90 & {$[0.54,1.45]$} & 0.6757 \\
\hline eGFR ( $1 \mathrm{ml} / \mathrm{min} / 1.73 \mathrm{~m}^{2}$ increse $)$ & 0.97 & {$[0.95,0.99]$} & 0.0037 \\
\hline eGFR slope (/year) & 0.99 & {$[0.94,1.03]$} & 0.5468 \\
\hline
\end{tabular}

H.R. Hazard ratio, C.I. Confidence interval, BMI body mass index, $B P$ blood pressure, $A C E$ angiotensin converting enzyme

predictive value of each formula because there is no accepted definition of variability in eGFR. Community-acquired acute kidney injury (AKI), which was excluded from the previous studies, was included in the present study. Exclusion of AKI may have compromised the results because it has been recognized as a risk factor for long-term renal outcome and mortality. 


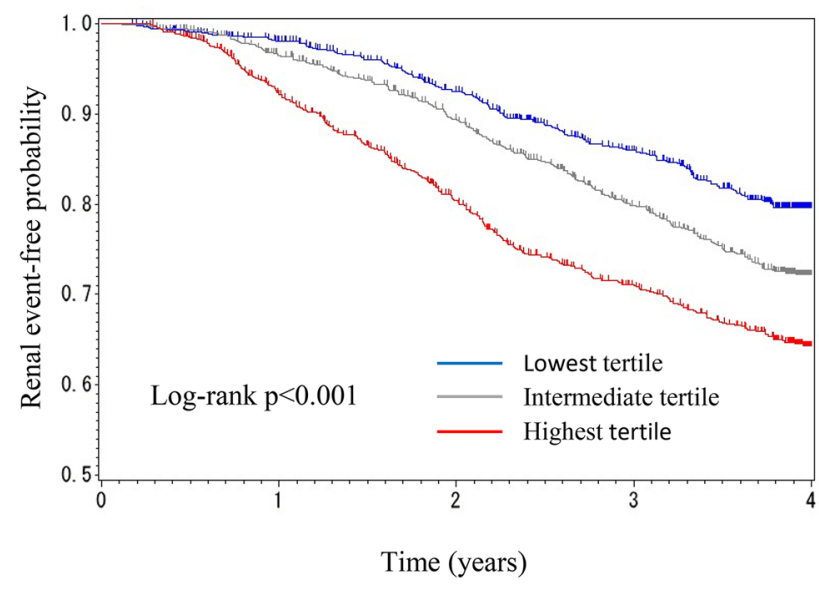

Fig. 3 Kaplan-Meier curve (time to ESKD or doubling of serum creatinine) by tertile of estimated glomerular filtration rate variability

In the setting of outpatient practice, visit-to-visit differences are commonly observed not only in the laboratory results but also in physical parameters such as blood pressure, heart rate, and body weight. The individual variation in eGFR may be affected by the volume status, blood pressure, deterioration (amelioration) of comorbidities, and modified medications. Of note, variations in blood pressure have been shown to be associated with not only the incidence of CVD and mortality [14], but also the decreased rate of renal function [15]. Recently, blood pressure variability was reported to be associated with the progression of carotid arteriosclerosis [16]. These findings could explain the results in the present study.

Variability in eGFR can be attributed to the loss of renal autoregulation of GFR depending on both the loss of functioning renal mass and atherosclerosis of renal arteries. We also analyzed the association between variability in eGFR and the composite renal outcome (ESKD or doubling of serum creatinine). Variability in eGFR was significantly and independently associated with the composite renal outcome in the multivariate-adjusted Cox proportional hazard model without the eGFR slope; however, it demonstrated no significant association in the analysis adjusted with the eGFR slope. These findings suggest that variability in eGFR may be caused predominantly by atherosclerosis rather than loss of renal mass because elevation of eGFR was not caused by the restoration of functioning nephrons in patients with CKD stage 3-5, which was getting progressively worse in most cases. In other words, patients with transient elevations in eGFR in the course of observation may have more severe systemic atherosclerotic changes in comparison to that in patients without transient elevation of eGFR.

The present study had some limitations. First, the cohort included only Asian patients; therefore, the findings may not be generalizable to other populations. Second, all patients were followed by nephrologists at established regional medical institutions. It is impossible for all Japanese patients to be treated in the same setting due to the limited number of nephrologists. A distinct number of CKD patients are supposed to be followed by cardiologists, diabetologists, and general physicians. Therefore, the findings of the present study may not be applicable to those patients. Third, the changes in prescribed medications, including diuretics and antihypertensives, were not recorded during the observational period. Modifying the doses of these drugs can affect the eGFR via volume status and blood pressure, which would result in greater variability in eGFR. Forth, urinary protein is a well-established risk factor for $\mathrm{CV}$ event and renal outcome; however, it was impossible to add urinary protein into the multivariate-adjusted analysis because of the many missing values (deficit rate 67.4\%).

\section{Conclusion}

In conclusion, the present study suggests that variability in eGFR is an independent predictor of hospitalization or death due to CV events among patients with CKD stage 3-5. Further study should be conducted to clarify the pathological basis linking the variability of eGFR to $\mathrm{CV}$ events.

Acknowledgements This study was conducted by principal investigators at the following medical centers: Yoshio Taguma M.D., Japan Community Health Care Organization Sendai Hospital (Miyagi); Yoshitaka Maeda M.D., JA Toride Medical Center (Ibaraki); Eiji Kusano M.D., Jichi Medical University (Tochigi); Kosaku Nitta M.D., Tokyo Women's Medical, University Hospital (Tokyo); Yasuhiro Komatsu M.D., St. Luke's International Hospital (Tokyo); Tadao Akizawa M.D., Showa University Hospital (Tokyo); Eriko Kinugasa M.D., Showa University Northern Yokohama Hospital (Kanagawa); Ashio Yoshimura M.D., Showa University Fujigaoka Hospital (Kanagawa); Hiroshige Ohashi M.D., Gifu Prefectural General Medical Center (Gifu); Yuzo Watanabe M.D., Kasugai, Municipal Hospital (Aichi); Kei Kurata M.D., Tosei General Hospital (Aichi); Yoshitaka Isaka M.D., Osaka University Hospital (Osaka); Yoshiharu Tsubakihara M.D., Osaka General Medical Center (Osaka); Masahito Imanishi M.D., Osaka City General Hospital (Osaka); Masaki Fukushima M.D., Kurashiki 
Table 4 Multivariate-adjusted Cox proportional hazard models for renal events

\begin{tabular}{|c|c|c|c|}
\hline & H.R. & 95\% C.I. & $p$ value \\
\hline \multicolumn{4}{|l|}{ Model 1} \\
\hline Gender (male vs female) & 2.00 & {$[1.68,2.39]$} & $<0.000$ \\
\hline Age (1-year increase) & 0.98 & {$[0.97,0.99]$} & $<0.000$ \\
\hline BMI & 1.02 & {$[1.00,1.04]$} & 0.122 \\
\hline History of heart disease & 0.84 & {$[0.66,1.04]$} & 0.120 \\
\hline Diabetes mellitus & 1.38 & {$[1.15,1.66]$} & 0.000 \\
\hline Diastoric BP & 0.98 & {$[0.97,0.99]$} & 0.000 \\
\hline Systoric BP & 1.03 & {$[1.02,1.03]$} & $<0.000$ \\
\hline Use of diuretics & 1.11 & {$[0.93,1.33]$} & 0.237 \\
\hline Use of ACE & 0.82 & {$[0.68,0.97]$} & 0.023 \\
\hline eGFR $\left(1 \mathrm{ml} / \mathrm{min} / 1.73 \mathrm{~m}^{2}\right.$ increse $)$ & 0.90 & {$[0.89,0.91]$} & $<0.000$ \\
\hline \multicolumn{4}{|l|}{ Model 2} \\
\hline eGFR variability (intermediate vs lowest) & 1.21 & {$[0.98,1.49]$} & 0.076 \\
\hline (Highest vs lowest) & 1.66 & {$[1.35,2.04]$} & $<0.000$ \\
\hline Gender (male vs female) & 2.02 & {$[1.70,2.41]$} & $<0.000$ \\
\hline Age (1-year increase) & 0.98 & {$[0.97,0.99]$} & $<0.000$ \\
\hline BMI & 1.02 & {$[1.00,1.04]$} & 0.082 \\
\hline History of heart disease & 0.81 & {$[0.64,1.01]$} & 0.068 \\
\hline Diabetes Mellitus & 1.29 & {$[1.07,1.55]$} & 0.0072 \\
\hline Diastoric BP & 0.98 & {$[0.97,0.99]$} & 0.0002 \\
\hline Systoric BP & 1.03 & {$[1.02,1.03]$} & $<0.000$ \\
\hline Use of diuretics & 1.05 & {$[0.88,1.26]$} & 0.5778 \\
\hline Use of ACE & 0.79 & {$[0.66,0.94]$} & 0.009 \\
\hline eGFR $\left(1 \mathrm{ml} / \mathrm{min} / 1.73 \mathrm{~m}^{2}\right.$ increse $)$ & 0.90 & {$[0.89,0.91]$} & $<0.000$ \\
\hline \multicolumn{4}{|l|}{ Model 3} \\
\hline eGFR variability (intermediate vs lowest) & 1.16 & {$[0.94,1.43]$} & 0.176 \\
\hline (Highest vs lowest) & 1.19 & {$[0.97,1.48]$} & 0.105 \\
\hline Gender (male vs female) & 1.91 & {$[1.59,2.29]$} & $<0.000$ \\
\hline Age (1-year increase) & 0.98 & {$[0.97,0.98]$} & $<0.000$ \\
\hline BMI & 1.02 & {$[0.99,1.04]$} & 0.199 \\
\hline History of heart disease & 0.75 & {$[0.60,0.95]$} & 0.016 \\
\hline Diabetes Mellitus & 1.21 & {$[0.99,1.46]$} & 0.0580 \\
\hline Diastoric BP & 0.98 & {$[0.97,0.99]$} & $<0.000$ \\
\hline Systoric BP & 1.02 & {$[1.02,1.03]$} & $<0.000$ \\
\hline Use of diuretics & 1.21 & {$[1.01,1.45]$} & 0.0338 \\
\hline Use of ACE & 0.75 & {$[0.63,0.90]$} & 0.0020 \\
\hline eGFR $\left(1 \mathrm{ml} / \mathrm{min} / 1.73 \mathrm{~m}^{2}\right.$ increse $)$ & 0.89 & {$[0.88,0.89]$} & $<0.000$ \\
\hline eGFR slope (/year) & 0.84 & {$[0.83,0.86]$} & $<0.000$ \\
\hline
\end{tabular}

H.R. Hazard ratio, C.I. Confidence nterval, BMI body mass index, BP blood pressure, ACE angiotensin converting enzyme
Central Hospital (Okayama); Hideki Hirakata M.D., Fukuoka Red Cross Hospital (Fukuoka); and Kazuhito Takeda M.D., Iizuka Hospital (Fukuoka).

Funding This study was supported by research funds with no restriction on publication from Kyowa Hakko Kirin Co., Ltd.

\section{Compliance with ethical standards}

Conflict of interest Consultant: E.I. (Kyowa Hakko Kirin). Research support grant: H.M. (Kyowa Hakko Kirin).

Informed consent Informed consent was obtained from all individual participants included in the study.

Open Access This article is distributed under the terms of the Creative Commons Attribution 4.0 International License (http://creativeco 
mmons.org/licenses/by/4.0/), which permits unrestricted use, distribution, and reproduction in any medium, provided you give appropriate credit to the original author(s) and the source, provide a link to the Creative Commons license, and indicate if changes were made.

\section{References}

1. Levey AS, Coresh J. Chronic kidney disease. Lancet. 2012;379:165-80.

2. Imai E, Horio M, Watanabe T, Iseki K, Yamagata K, Hara S, et al. Prevalence of chronic kidney disease in the Japanese general population. Clin Exp Nephrol. 2009;13:621-30.

3. Go AS, Chertow GM, Fan D, McCulloch CE, Hsu C. Chronic kidney disease and the risks of death, cardiovascular events, and hospitalization. N Engl J Med. 2004;2004; 351:1296-305.

4. van der Velde M, Matsushita K, Coresh J, Astor BC, Woodward $\mathrm{M}$, Levey AS, et al. Lower estimated glomerular filtration rate and higher albuminuria are associated with all-cause and cardiovascular mortality. A collaborative meta-analysis of high-risk population cohorts. Kidney Int. 2011;79:1341-52.

5. Astor BC, Matsushita K, Gansevoort RT, van der Velde M, Woodward M, Levey AS, et al. Lower estimated glomerular filtration rate and higher albuminuria are associated with mortality and end-stage renal disease. A collaborative meta-analysis of kidney disease population cohorts. Kidney Int. 2011;79:1331-40.

6. Al-Aly Z, Zeringue A, Fu J, Rauchman MI, McDonald JR, ElAchkar TM, et al. Rate of kidney function decline associates with mortality. J Am Soc Nephrol. 2010;21:1961-9.

7. Perkins RM, Bucaloiu ID, Kirchner HL, Ashouian N, Hartle JE, Yahya T. GFR decline and mortality risk among patients with chronic kidney disease. Clin J Am Soc Nephrol. 2011;6:1879-86.

8. Turin TC, Coresh J, Tonelli M, Stevens PE, de Jong PE, Farmer $\mathrm{CK}$, et al. One-year change in kidney function is associated with an increased mortality risk. Am J Nephrol. 2012;36:41-9.
9. Al-Aly Z, Balasubramanian S, McDonald JR, Scherrer JF, O'Hare AM. Greater variability in kidney function is associated with an increased risk of death. Kidney Int. 2012;82:1208-14.

10. Perkins RM, Tang X, Bengier AC, Kirchner HL, Bucaloiu ID. Variability in estimated glomerular filtration rate is an independent risk factor for death among patients with stage 3 chronic kidney disease. Kidney Int. 2012;82:1332-8.

11. Imai E, Matsuo S, Makino $H$, Watanabe $T$, Akizawa $T$, Nitta $K$, et al. Chronic kidney disease Japan Cohort (CKD-JAC) study: design and methods. Hypertens Res. 2008;31:1101-7.

12. Matsuo S, Imai E, Horio M, Yasuda Y, Tomita K, Nitta K, et al. Revised equations for estimated GFR from serum creatinine in Japan. Am J Kidney Dis. 2009;53:982-92.

13. Imai E, Matsuo S, Makino H, Watanabe T, Akizawa T, Nitta K, et al. Chronic Kidney Disease Japan cohort study: baseline characteristics and factors associated with causative diseases and renal function. Clin Exp Nephrol. 2010;14:558-70.

14. Wang J, Shi X, Ma C, Zheng H, Xiao J, Bian H, et al. Visit-to-visit blood pressure variability is a risk factor for all-cause mortality and cardiovascular disease. J Hypertens. 2017;35:10-7.

15. Chia YC, Lim HM, Ching SM. Long-term visit-to-visit blood pressure variability and renal function decline in patients with hypertension over 15 years. J Am Heart Assoc. 2016;5:e003825.

16. Wu D, Li C, Chen Y, Xiong H, Tian X, Wu W, et al. Influence of blood pressure variability on early carotid atherosclerosis in hypertension with and without diabetes. Medicine (Baltimore). 2016;95:e3864.

Publisher's Note Springer Nature remains neutral with regard to jurisdictional claims in published maps and institutional affiliations. 